\title{
Effect of color reconnection and rope formation on resonance production in p-p collisions in Pythia 8
}

\author{
Ankita Goswami, Ranjit Nayak, Basanta Kumar Nandi, Sadhana Dash ${ }^{\mathrm{a}}$ \\ Indian Institute of Technology Bombay, Mumbai 400076, India
}

Received: 6 April 2021 / Accepted: 5 October 2021 / Published online: 10 November 2021

(C) The Author(s) 2021

\begin{abstract}
Resonance production in proton-proton collisions at $\sqrt{s}=7 \mathrm{TeV}$ and $13 \mathrm{TeV}$ have been investigated using a Pythia 8 event generator within the framework of microscopic processes including color reconnection and rope hadronization. Specifically, the observable effects of different modes of color reconnections on the ratio of yields of mesonic and baryonic resonances with respect to their stable counterpart have been explored as a function of mean charged particle multiplicity. A suppression in the ratio is observed as a function of the mean number of charged particles for mesonic resonances. The $\phi / \mathrm{K}$ and $\phi / \pi$ ratios show an enhancement for high-multiplicity events due to enhanced production of strange quarks via the microscopic process of rope hadronization in the partonic phase. The mechanism of the hadronization of color ropes together with the quarkgluon plasma (QCD)-based color reconnection of partons predicted an enhancement in the ratio for baryonic resonances to non-resonance baryons having similar quark content. The yield ratios of resonances are found to be independent of the collision energy and strongly dependent on event activity.
\end{abstract}

\section{Introduction}

Proton-proton ( $\mathrm{p}-\mathrm{p}$ ) collisions have been studied extensively as a reference for the study of medium-induced phenomena in heavy ion collisions. However, recent measurements performed in high-multiplicity $\mathrm{p}-\mathrm{p}$ and proton-lead $(\mathrm{p}-\mathrm{Pb})$ collisions at the Large Hadron Collider (LHC) exhibited features such as strangeness enhancement [1], long-range ridge-like structure in the near side of two-particle correlations [2], and collectivity effects [3] which are similar to those observed in heavy ion collisions. These interesting observations have inspired the investigation of collectivity-driven features in small systems (like $\mathrm{p}-\mathrm{p}$ ) that are currently actively pursued.

a e-mail: Sadhana.Dash@cern.ch (corresponding author)
In high-energy experiments at the Relativistic Heavy Ion Collider (RHIC) and LHC, the bulk properties of the medium formed due to collision of heavy ions have been widely studied to comprehend the conditions of the late hadronic phase together with the effects of collective expansion from the initial stages. The hadronic resonances whose lifetimes are shorter than or comparable to the time scale for evolution of the fireball are extremely sensitive to probe the properties of the dense medium [4]. This might lead to modifications in their production rate and characteristic properties (such as in vacuo mass and width) due to dynamical interactions with the surrounding matter. The fraction of resonances that decay before the kinetic freeze-out (vanishing elastic interactions) are less likely to be reconstructed due to elastic scattering of the decay products with the surrounding medium, known as the resonance re-scattering effect. Alternatively, the yield might increase in the hadronic phase via interactions known as resonance regeneration and can partially or fully compensate the yield loss due to the rescattering effect. The observation of suppression of the relative yield ratios of $K^{* 0} / \mathrm{K}[5,6]$ and $\Lambda(1520) / \Lambda$ [7] with centrality was attributed to the interplay of the mediuminduced effects in the hadronic phase. The EPOS model estimations on hadronic resonances also described the suppression of $\mathrm{K}^{* 0} / \mathrm{K}$ in central $\mathrm{Pb}-\mathrm{Pb}$ collisions due to interactions in the extended hadronic phase $[8,9]$. However, recent observation of suppression of $\mathrm{K}^{* 0} / \mathrm{K}$ in high-multiplicity $\mathrm{p}-\mathrm{p}$ collisions [10] has triggered discussions regarding the role of other microscopic mechanisms present in the partonic phase. The $\mathrm{p}-\mathrm{p}$ system, due to its smallness, is not expected to have a medium or active hadronic phase to account for the observed suppression. The study of such small systems provides insights into the degree of similarity of the hadronization process to that present in larger systems, and can shed light on the contributions from the initial partonic phase. It was recently reported that microscopic processes such as color reconnections and rope hadroniza- 
tion were able to describe the observation of strangeness enhancement [11,12], near-side long-range ridge-like correlations [13], and flow-like effects [3] in p-p collisions at LHC energies. In a recent study on $\mathrm{p}-\mathrm{p}$ collisions using the Pythia 8 generator [15], the QCD-based color reconnection mechanism was able to reproduce the suppression trend in the $\mathrm{K}^{* 0} / \mathrm{K}$ ratio as a function of event activity [16]. Color reconnections $(\mathrm{CR})$ refer to the mechanism of formation of hadronizing strings between the outgoing partons regardless of their formation history. The Pythia 8 model implements the mechanism on the basis of minimization of the total string potential energy stored in these strings. The final outgoing partons generally tend to be color-correlated with the partons which are nearer to them in momentum space, resulting in minimizing the string length. Consequently, the total potential energy stored in these strings is also minimized. The details of the color reconnection mechanism can be found in Refs. [17,18]. In Pythia 8, the QCD-based color reconnection [(Color reconnections $((1)]$ allows the formation of junctions where three color lines join and can result in an enhancement of baryons. For high-multiplicity $\mathrm{p}-\mathrm{p}$ collisions, it may happen that several strings are very close to each other and thus mutually interact to form color ropes. These ropes have a larger effective string tension and are likely to fragment into more strange quarks and di-quarks, which leads to enhanced production of baryons and strange hadrons $[19,20]$. The suppression of mesonic resonances using Pythia 8 was attributed to the mechanism of QCD-based color reconnection [16]. The CR lead to the formation of shorter string lengths which are more likely to fragment into less massive particles. As resonances are massive, the resonance-to-nonresonance ratio decreases for high-multiplicity events where the effect of color reconnections is more noticeable. In this work, the study of resonance-to-non-resonance ratios is carried out to investigate the effects of $\mathrm{CR}$ and rope hadronization on mesonic and baryonic resonances using Pythia 8.

\section{Pythia 8 simulations}

This study is based on 20 million events of inelastic $\mathrm{p}-\mathrm{p}$ collisions generated at $\sqrt{s}=7 \mathrm{TeV}$ and $13 \mathrm{TeV}$ each using the Pythia 8 event generator. The Monash 2013 tune [21] was used to simulate the events with multi-partonic interactions (MPI), rope hadronization ( $\mathrm{RH}$ ), and two different modes of color reconnections [color reconnections $(0)$ and color reconnections (1)]. The color reconnections (0) refers to the MPI-based reconnection scheme where lower $p_{T}$ partons are added to higher $p_{T}$ partons to minimize the string length. The color reconnections (1) reconnection scheme is the QCD-based model based on minimizing the string length, where the QCD multiplets can produce triplets and junctions which produce more baryons. The production of various mesonic resonances including $\mathrm{K}^{*}, \phi, \eta^{\prime}$, and $\rho$, as well as the baryonic resonances such as $\Delta^{+}, \Sigma^{* 0}$, and $\Xi^{* 0}$, specifically the ratios of their yield to the yield of their stable counterpart (having same quark content), is studied as a function of event activity. The ratios of the considered resonance particles with their stable counterpart were obtained for $|\eta|<0.5$ for different configurations. The configurations involved various combinations of the different modes of color reconnections with (and without) the formation of ropes. As the number of MPIs is strongly correlated with the observed charged particle multiplicity, the effect of various mechanisms on the resonance-to-non-resonance ratio is studied as a function of mean charged particle multiplicity $\left(<N_{\mathrm{ch}}>\right)$. The observed variation with respect to $\left\langle N_{\mathrm{ch}}>\right.$ is expected to yield a similar trend as a function of the number of multi-partonic interactions (event activity) and is advantageous, as the predictions can be directly compared with experimental results. Table 1 shows the various configurations used for the analysis.

\section{Mesonic resonances}

Figure 1 shows the yield ratios of $\mathrm{K}^{* 0} / \mathrm{K}$ as a function of $<N_{\text {ch }}>$ for $\mathrm{p}-\mathrm{p}$ collisions at $\sqrt{s}=7 \mathrm{TeV}$ and $13 \mathrm{TeV}$. In the left panel of Fig. 1, the ratio of $\mathrm{K}^{*} / \mathrm{K}$ shows a gradual suppression for high-multiplicity events for QCD-based color reconnection [color reconnections (1)] when compared to the scenario where there is no color reconnection or there is only MPI-based color reconnection [color reconnections (0)]. The suppression trend is qualitatively similar to that observed in data as measured by the ALICE experiment [22,23]. However, the values slightly overestimate the measured data for $7 \mathrm{TeV}$, while the agreement is good for $13 \mathrm{TeV}$. The values obtained after enabling RH are similar to those of color reconnections (1), and its effect is not significant. The middle panel and the lower panel compare the ratio for $\mathrm{p}-\mathrm{p}$ collisions at $\sqrt{s}=7 \mathrm{TeV}$ and $13 \mathrm{TeV}$. There is no effect of increasing the beam energy on the ratios, indicating that the suppression is comparable for similar event activity. The suppression is similar for both color reconnections -off and color reconnections (0) mode. The enabling of CR leads to the production of a large number of shorter strings, and the effect is more pronounced for the color reconnections (1) scheme [16]. Therefore, the production of massive $\mathrm{K}^{* 0}$ is suppressed compared to kaons in high-multiplicity events, as shorter strings fragment to less massive particles. The formation of ropes in combination with the color reconnections (1) gives similar suppression, as $\mathrm{RH}$ leads to enhanced production of strange hadrons and baryons. The effect of $\mathrm{RH}$ is not expected to be visible for $\mathrm{K}^{* 0} / \mathrm{K}$, as the quark content is same for both the particles. We further investigated the yield ratio of $\eta^{\prime} / \pi$ as a function of mean charged particle multiplicity for $\mathrm{p}-\mathrm{p}$ 
Table 1 A list of different configurations (processes) used in Pythia 8 for the analysis. MPI, multi-partonic interactions; $\mathrm{CR}$, color reconnection; $\mathrm{RH}$, rope hadronization

\begin{tabular}{ll}
\hline Different configurations & Definitions of different settings \\
\hline MPI on CR off & With MPI and without CR and RH \\
MPI on CR (0) on & With MPI and with MPI based CR and without RH \\
MPI on CR (1) on & With MPI and with QCD based CR and without RH \\
MPI on CR (1) on RH on & With MPI and with QCD based CR and with RH \\
MPI on CR off RH on & With MPI and with RH and without CR \\
\hline
\end{tabular}

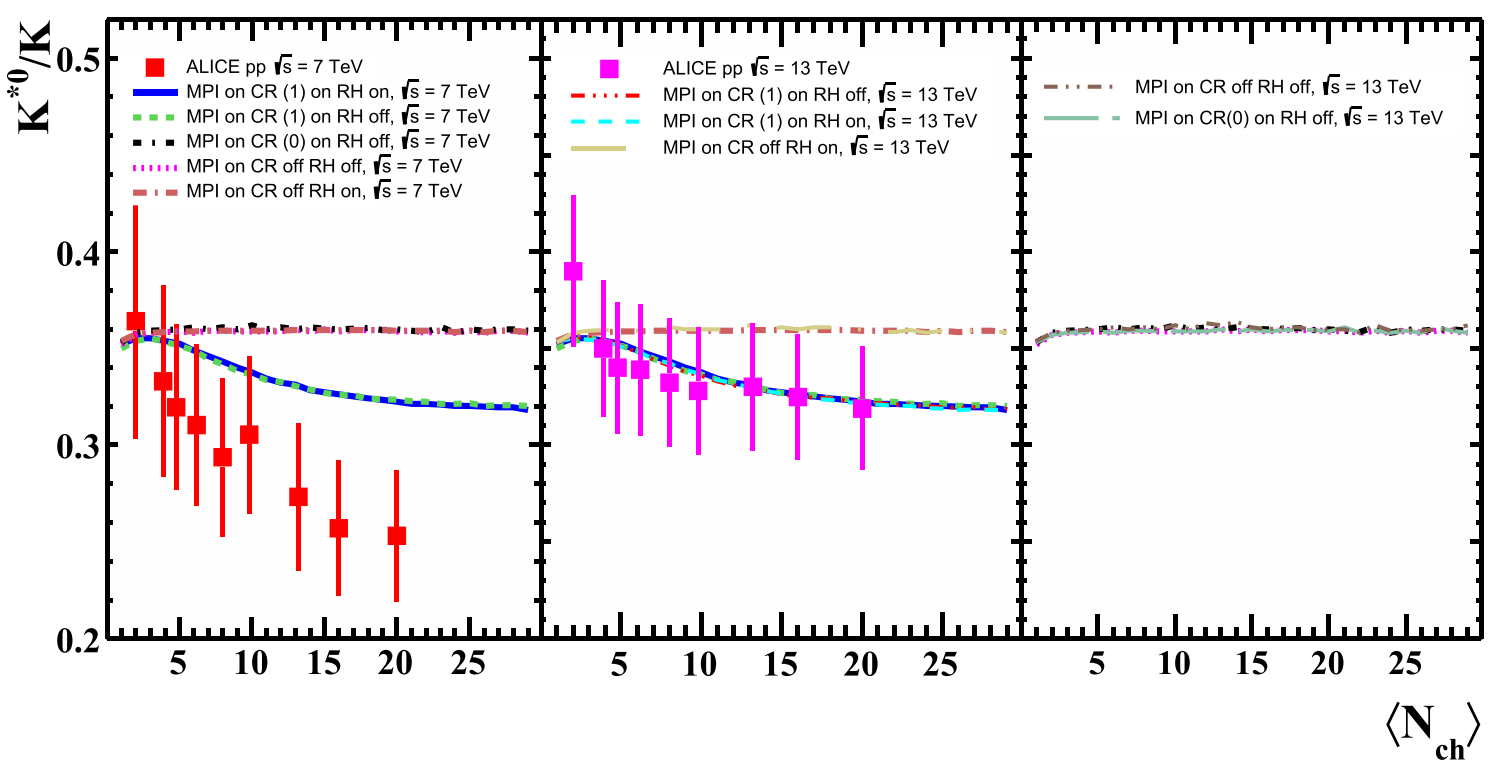

Fig. $1 p_{T}$-integrated ratio of $\mathrm{K}^{* 0} / \mathrm{K}$ as a function of $<N_{\mathrm{ch}}>$ for $\mathrm{p}-\mathrm{p}$ collisions. (Left panel) The ratios are compared for two different modes of color reconnections [CR-off, CR(0) and CR(1)] with (and without) RH in $\mathrm{p}-\mathrm{p}$ collisions at $\sqrt{s}=7 \mathrm{TeV}$. (Middle panel) The ratios are compared for $\mathrm{p}-\mathrm{p}$ collisions at $\sqrt{s}=7 \mathrm{TeV}$ and $13 \mathrm{TeV}$ for CR-off,

collisions at $\sqrt{s}=7 \mathrm{TeV}$ and $13 \mathrm{TeV}$ in Fig. 2. Both of the considered particles in the ratio are spin- 0 particles, with $\eta^{\prime}$ being heavier than $\pi$. A similar trend as $\mathrm{K}^{* 0} / \mathrm{K}$ suppression is observed which can be attributed to the production of pions being favored more than $\eta^{\prime}$ due to formation and fragmentation of shorter strings. This observation can be attributed to the mechanism of color reconnection. The variation in $\rho^{0} / \pi$ with multiplicity is shown in Fig. 3. Surprisingly, greater suppression in the yield ratio is observed with the onset of RH in combination with CR(1) for high-multiplicity events. Since $\rho^{0}$ is not a strange hadron, it seems that enabling RH further reduces its production in favor of other strange hadrons or baryons in high-multiplicity events. However, a similar suppression was not seen for $\mathrm{K}^{* 0} / \mathrm{K}$ on enabling $\mathrm{RH}$, as both the particles are strange hadrons. The yield ratios of $\phi / \mathrm{K}$ and $\phi / \pi$ were also considered as a function of event multiplicity as illustrated in Figs. 4 and 5, respectively. The figure also compares the dependence of the ratios as a function of $N_{\mathrm{ch}}$ as measured by the ALICE experiment for $\mathrm{p}-\mathrm{p}$ collisions at $\sqrt{s}=7 \mathrm{TeV}$ and $13 \mathrm{TeV}[22,23]$. $\phi$ is a spin- 1 resonance par-
$\mathrm{CR}(0)$ and $\mathrm{CR}(1)$ with RH. (Right panel) Comparison of ratios for $\mathrm{p}-\mathrm{p}$ collisions at $\sqrt{s}=7 \mathrm{TeV}$ and $13 \mathrm{TeV}$ for two other modes, namely CR-off and $\mathrm{CR}(0)$ without $\mathrm{RH}$. The solid squares show the multiplicity dependence of $\mathrm{K}^{* 0} / \mathrm{K}$ for $\mathrm{p}-\mathrm{p}$ collisions at $\sqrt{s}=7 \mathrm{TeV}$ and $13 \mathrm{TeV}$ as obtained by the ALICE experiment $[22,23]$

ticle with a relatively longer lifetime. Although $\phi$ consists of $s \bar{s}$ quarks, the yield ratios are enhanced due to the production of more strange (and anti-strange) quark pairs due to the formation of color ropes. The enhancement is more pronounced for color reconnections (1) in combination with $\mathrm{RH}$, and the enhancement in the yield ratios is independent of event activity and energies. It can be observed that the ratios are in good agreement with the measured data with the onset of both color reconnections (1) and RH. It is interesting to note that the ratio for color reconnections (1) is consistently smaller than color reconnections ( 0 ) and CR-off. This can be attributed to the production of lower mass particles because color reconnections (1) favors the formation of shorter mass strings.

Figure 6 shows the comparison of $\mathrm{K}^{* 0} / \mathrm{K}, \phi / \mathrm{K}$, and $\phi / \pi$ ratio obtained from Pythia 8 [with color reconnections(1) and $\mathrm{RH}$ on] with the recent ALICE measurements in $\mathrm{p}-\mathrm{p}$ collisions at $\sqrt{s}=7 \mathrm{TeV}$. The open circles show the yield ratios obtained from Pythia 8, while the open squares represent the measured data. The values obtained from Pythia 8 are in good 


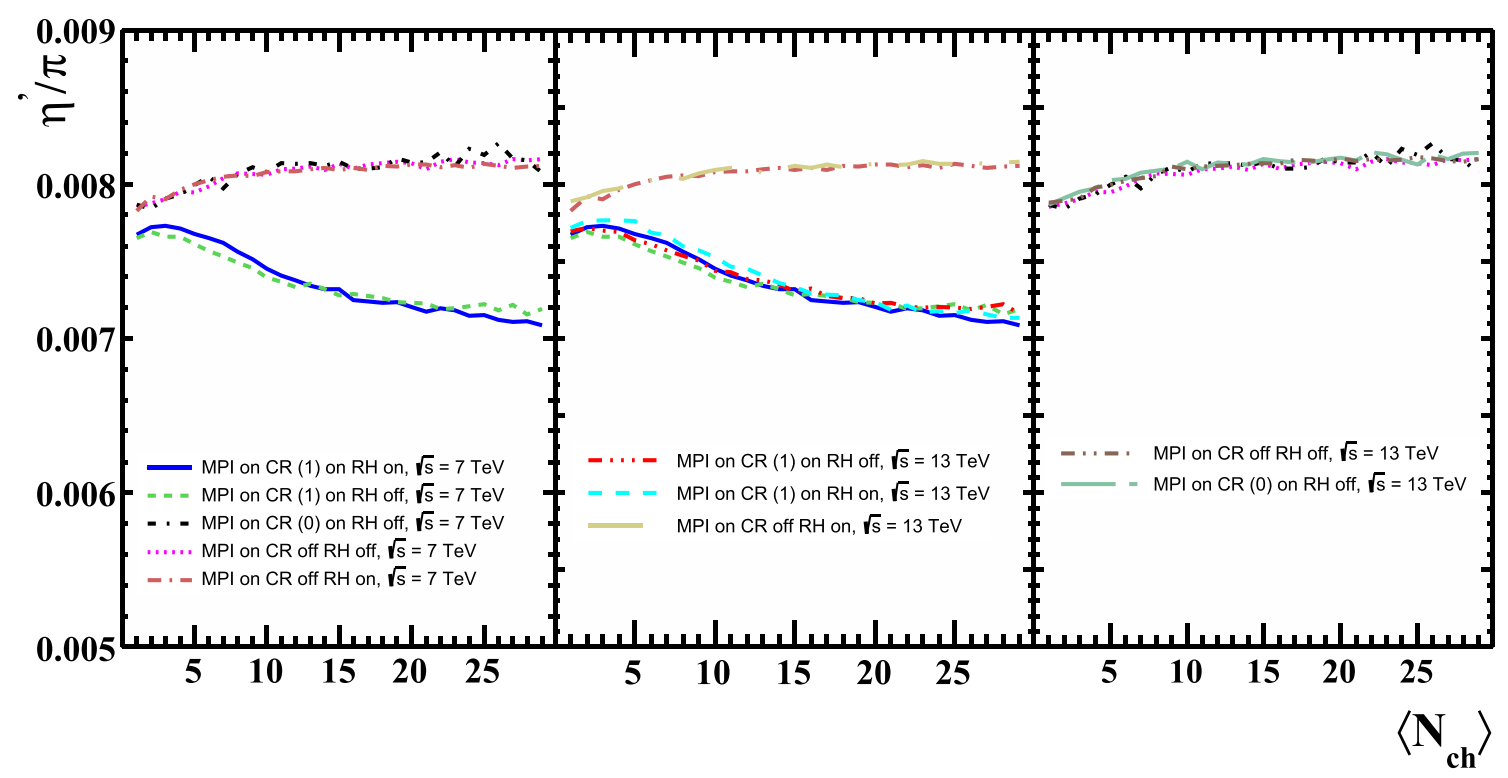

Fig. $2 p_{T}$-integrated ratio of $\eta^{\prime} / \pi$ as a function of $<N_{\mathrm{ch}}>$ for $\mathrm{p}-\mathrm{p}$ collisions. (Left panel) The ratios are compared for two different modes of color reconnections [CR-off, $\mathrm{CR}(0)$ and $\mathrm{CR}(1)$ ] with (and without) $\mathrm{RH}$ in $\mathrm{p}-\mathrm{p}$ collisions at $\sqrt{s}=7 \mathrm{TeV}$. (Middle panel) The ratios are compared for $\mathrm{p}-\mathrm{p}$ collisions at $\sqrt{s}=7 \mathrm{TeV}$ and $13 \mathrm{TeV}$ for CR-off, $\mathrm{CR}(0)$ and $\mathrm{CR}(1)$ with RH. (Right panel) Comparison of ratios for $\mathrm{p}-\mathrm{p}$ collisions at $\sqrt{s}=7 \mathrm{TeV}$ and $13 \mathrm{TeV}$ for two other modes, namely CR-off and $\mathrm{CR}(0)$ without RH

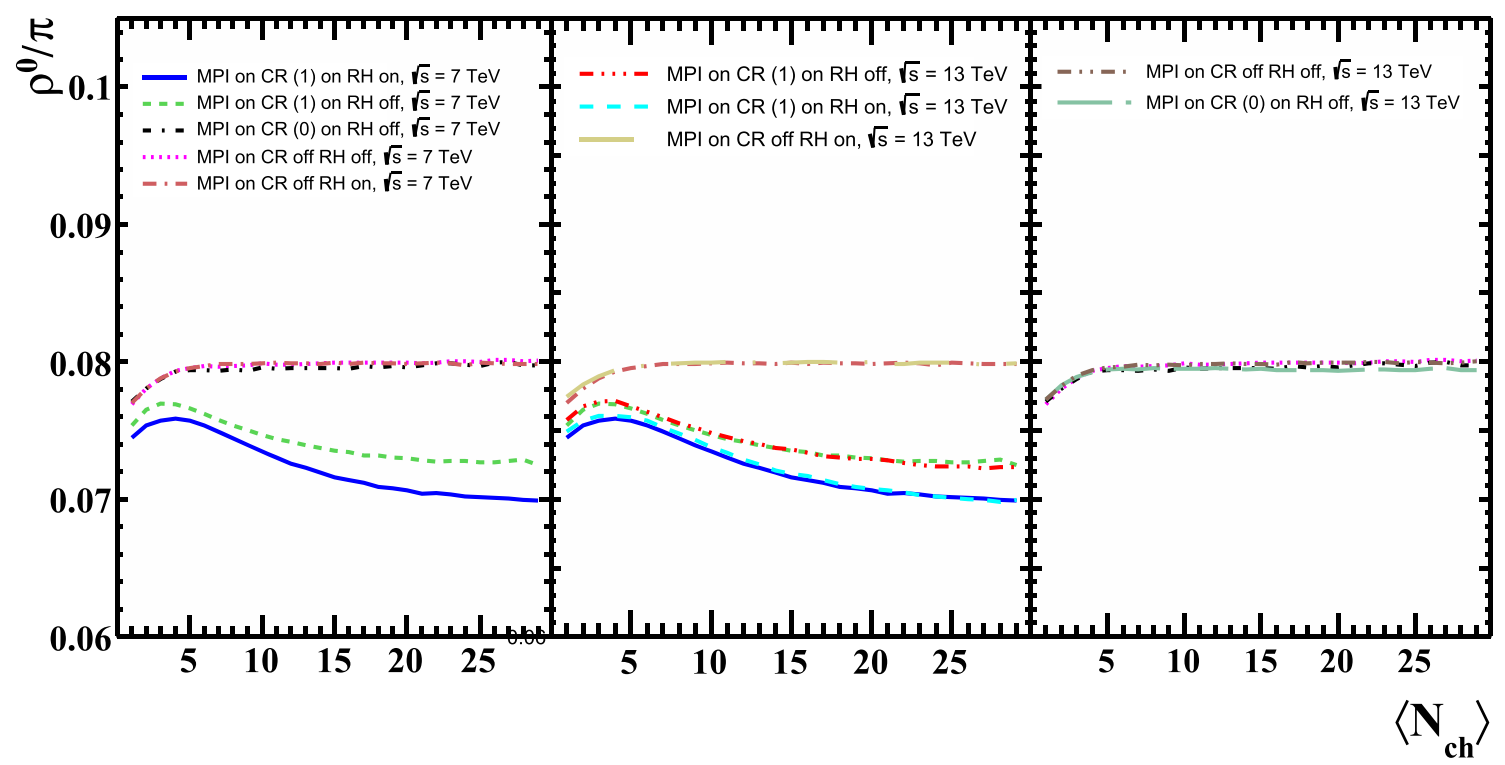

Fig. $3 p_{T}$-integrated ratio of $\rho^{0} / \pi$ as a function of $<N_{\mathrm{ch}}>$ for $\mathrm{p}-\mathrm{p}$ collisions. (Left panel) The ratios are compared for two different modes of CR [CR-off, CR(0) and CR(1)] with (and without) RH in p-p collisions at $\sqrt{s}=7 \mathrm{TeV}$. (Middle panel) The ratios are compared for $\mathrm{p}-\mathrm{p}$

agreement with the measured data within the uncertainties, which indicates that microscopic processes like RH and color reconnection play an important role in describing the particle production mechanism at high energies. collisions at $\sqrt{s}=7 \mathrm{TeV}$ and $13 \mathrm{TeV}$ for CR-off, $\mathrm{CR}(0)$ and $\mathrm{CR}(1)$ with RH. (Right panel) Comparison of ratios for $\mathrm{p}-\mathrm{p}$ collisions at $\sqrt{s}=7$ $\mathrm{TeV}$ and $13 \mathrm{TeV}$ for two other modes, namely CR-off and CR( 0 ) without $\mathrm{RH}$

\section{Baryonic resonances}

The particle ratios involving the baryonic resonances are also investigated by studying the $\Delta^{+} / p, \Sigma^{0 *} / \Lambda, \Sigma^{0 *} / \Sigma^{0}$, and $\Xi^{0 *} / \Xi^{0}$. Figures 7 and 8 show the variation in the yield ratios of $\Sigma^{0 *} / \Sigma^{0}$ and $\Sigma^{0 *} / \Lambda$ with respect to $<N_{\mathrm{ch}}>$. It is 


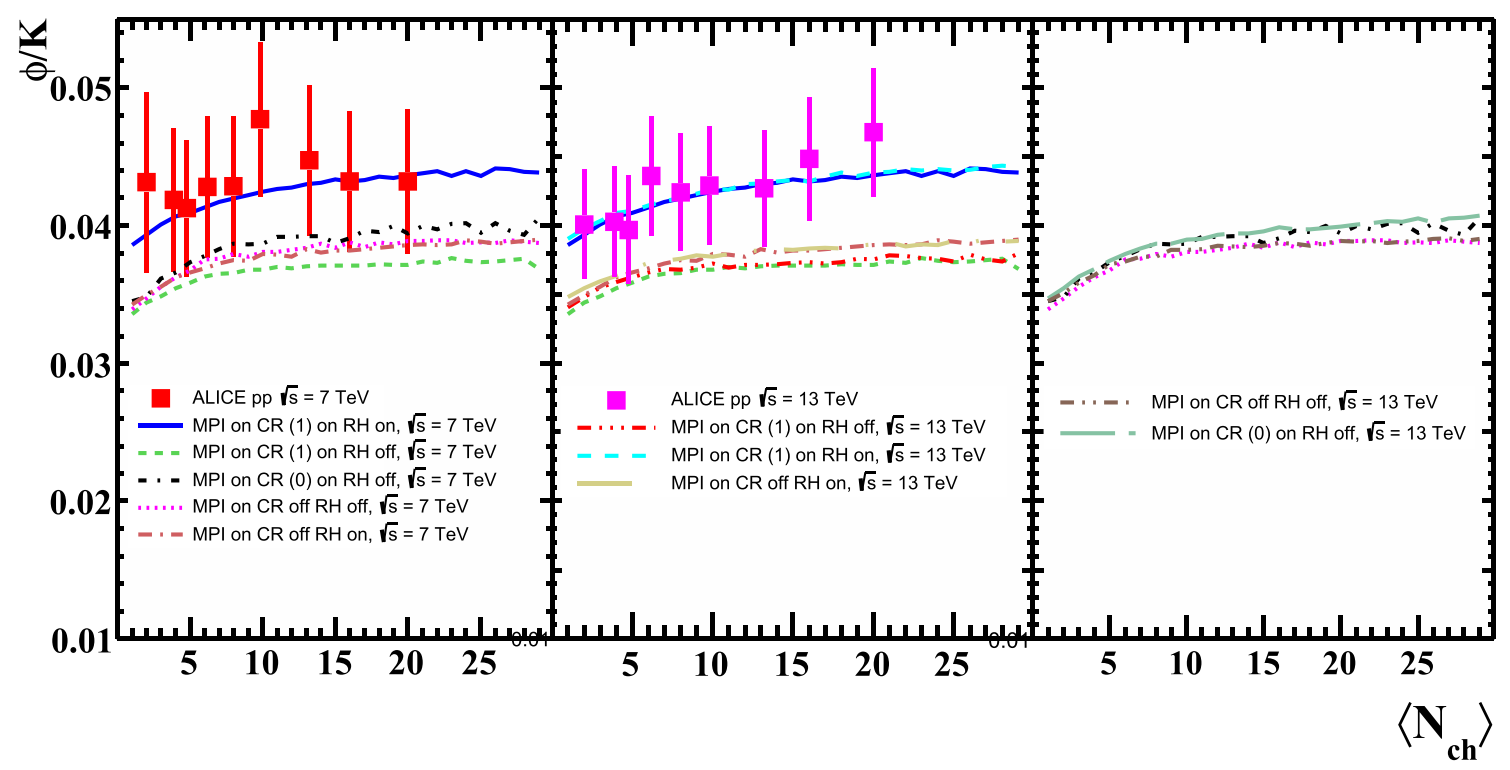

Fig. $4 p_{T}$-integrated ratio of $\phi / \mathrm{K}$ as a function of $<N_{\mathrm{ch}}>$ in $\mathrm{p}-\mathrm{p}$ collisions. (Left panel) The ratios are compared for two different modes of CR [CR-off, CR(0) and CR(1)] with (and without) RH in p-p collisions at $\sqrt{s}=7 \mathrm{TeV}$. (Middle panel) The ratios are compared for $\mathrm{p}-\mathrm{p}$ collisions at $\sqrt{s}=7 \mathrm{TeV}$ and $13 \mathrm{TeV}$ for CR-off, CR(0) and CR(1) with
RH.(Right panel) Comparison of ratios for $\mathrm{p}-\mathrm{p}$ collisions at $\sqrt{s}=7$ $\mathrm{TeV}$ and $13 \mathrm{TeV}$ for two other modes, namely CR-off and CR(0) without RH. The solid squares show the multiplicity dependence of $\phi / \mathrm{K}$ for $\mathrm{p}-\mathrm{p}$ collisions at $\sqrt{s}=7 \mathrm{TeV}$ and $13 \mathrm{TeV}$ as obtained by the ALICE experiment $[22,23]$

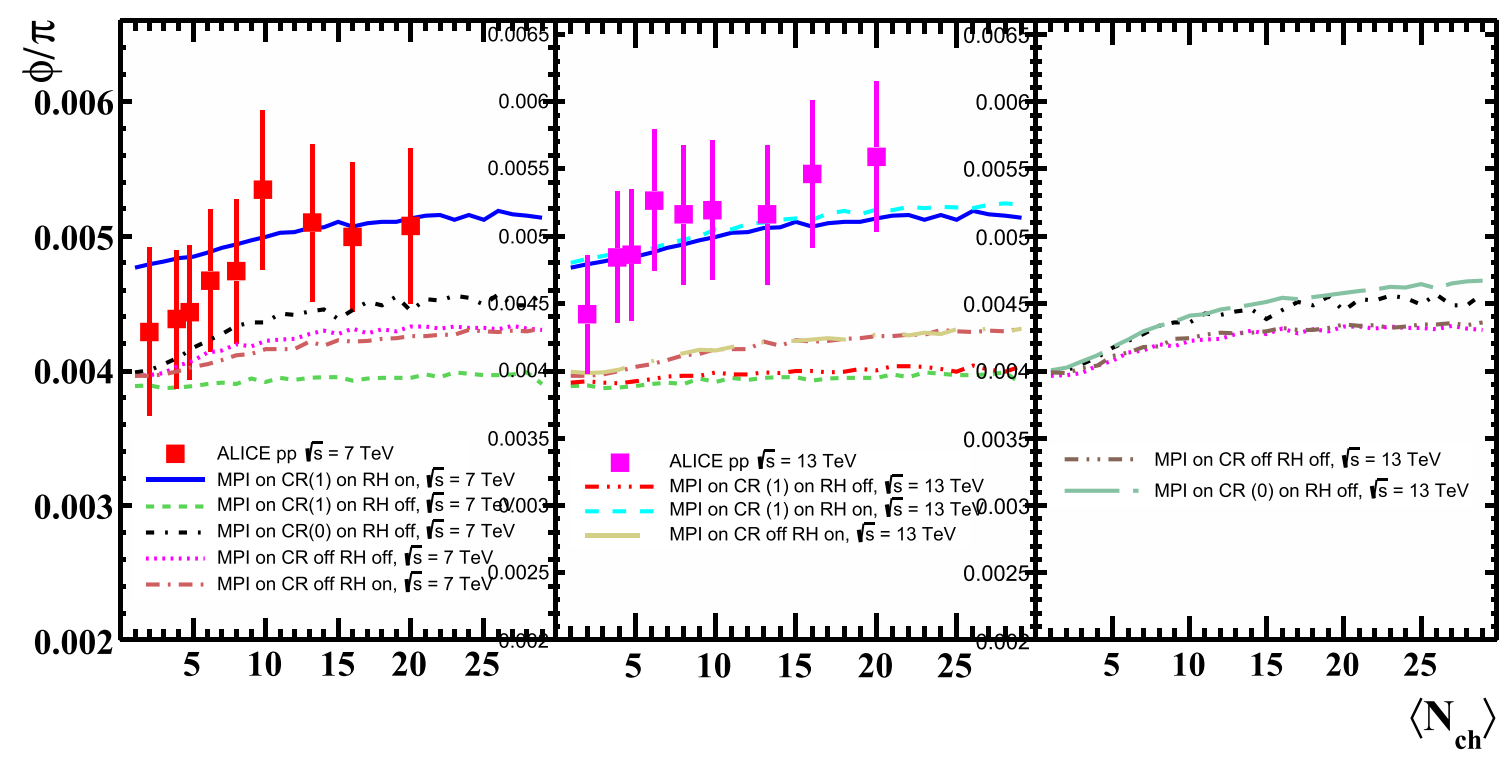

Fig. $5 p_{T}$-integrated ratio of $\phi / \pi$ as a function of $<N_{\text {ch }}>$ in $\mathrm{p}-\mathrm{p}$ collisions. (Left panel) The ratios are compared for two different modes of CR [CR-off, CR(0) and CR(1)] with (and without) RH in p-p collisions at $\sqrt{s}=7 \mathrm{TeV}$. (Middle panel) The ratios are compared for $\mathrm{p}-\mathrm{p}$ collisions at $\sqrt{s}=7 \mathrm{TeV}$ and $13 \mathrm{TeV}$ for CR-off, $\mathrm{CR}(0)$ and $\mathrm{CR}(1)$ with
RH. (Right panel) Comparison of ratios for $\mathrm{p}-\mathrm{p}$ collisions at $\sqrt{s}=7$ $\mathrm{TeV}$ and $13 \mathrm{TeV}$ for two other modes, namely CR-off and CR(0) without RH. The solid squares show the multiplicity dependence of $\phi / \mathrm{K}$ and $\phi / \pi$ for $\mathrm{p}-\mathrm{p}$ collisions at $\sqrt{s}=7 \mathrm{TeV}$ and $13 \mathrm{TeV}$ as obtained by the ALICE experiment [22,23] interesting to observe the evolution of $\Sigma^{0 *} / \Sigma^{0}$ and $\Sigma^{0 *} / \Lambda$ with event activity, as the particles in the denominator have identical quark content but differ in spin. $\Sigma^{0}$ belongs to the isospin triplet, while $\Lambda^{0}$ is a singlet. We observe an enhancement in the ratios of $\Sigma^{0 *} / \Sigma^{0}$ and $\Sigma^{0 *} / \Lambda$ with color recon- nections(1), but the enhancement is more pronounced in the case of RH for the former, while no visible effect is seen for the latter. This shows that production of baryons belonging to a higher-spin multiplet is favored when $\mathrm{RH}$ is switched on. This leads to more production of $\Sigma^{0 *}$ compared to $\Sigma^{0}$. 


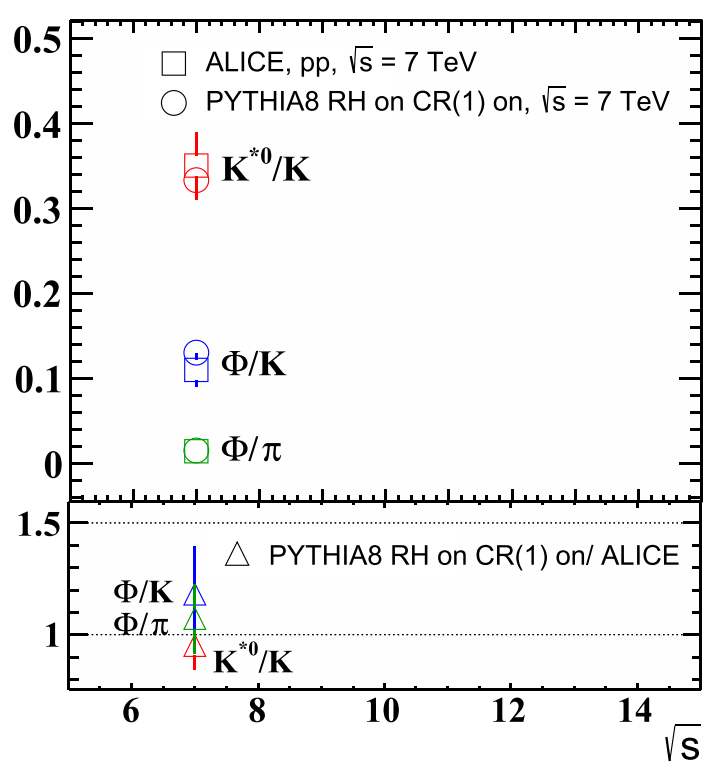

Fig. 6 The $p_{T}$-integrated ratio as a function of charged particle multiplicity in proton-proton collisions at $7 \mathrm{TeV}$ [5]

However, the effect is not seen for $\Sigma^{* 0} / \Lambda$. The observation can be attributed to the difference in their spin and isospin quantum number. The effect has no significant dependence on energies, as can be clearly seen in the middle and right panels of Fig. $7 \mathrm{a}, \mathrm{b}$. Figure 9 shows the $\Delta^{+} / p$ ratio as a function of event activity. $\Delta^{+}$is an interesting baryon, as it has an extremely short lifetime, it is a non-strange baryon with higher spin (3/2), and it has same quark content as the proton. They differ in mass and spin. The ratio is observed to increase with event activity when color reconnections(1) is enabled and RH has no observable effect on the ratios. This observed enhancement is in agreement with the effects of color reconnections (1), as color reconnections (1) produces more baryons of higher spin. The enabling of RH has no effect on enhancement, as both the particles are non-strange particles. Furthermore, the $\Xi^{0 *} / \Xi^{0}$ ratio as shown in Fig. 10 was also investigated, as $\Xi$ is a doubly strange baryon. The dashed line shows the $\Xi^{0 *} / \Xi^{0}$ ratio as measured by the ALICE experiment for integrated multiplicity [23]. Both particles have same quark content, but differ in mass and spin. The effect of color reconnections(1) leads to an enhancement because of favorable production of higher-spin baryons. Additionally, the color rope formation increases the production of strange quarks and favors the strange baryon production with higher spin, which is responsible for further enhancement in the ratio with RH. Collisional energy independence is also observed for both the ratios as illustrated in the middle and right panels of Figs. 9 and 10.

For all the resonances, both mesonic and baryonic, the effect of RH was also studied without the mechanism of color reconnections, and it was observed that it has no effect on the ratios, which confirms that color reconnection plays the most important role in the observed suppression (or enhancement) of the resonance-to-non-resonance ratios. These results serve as a baseline for future measurements

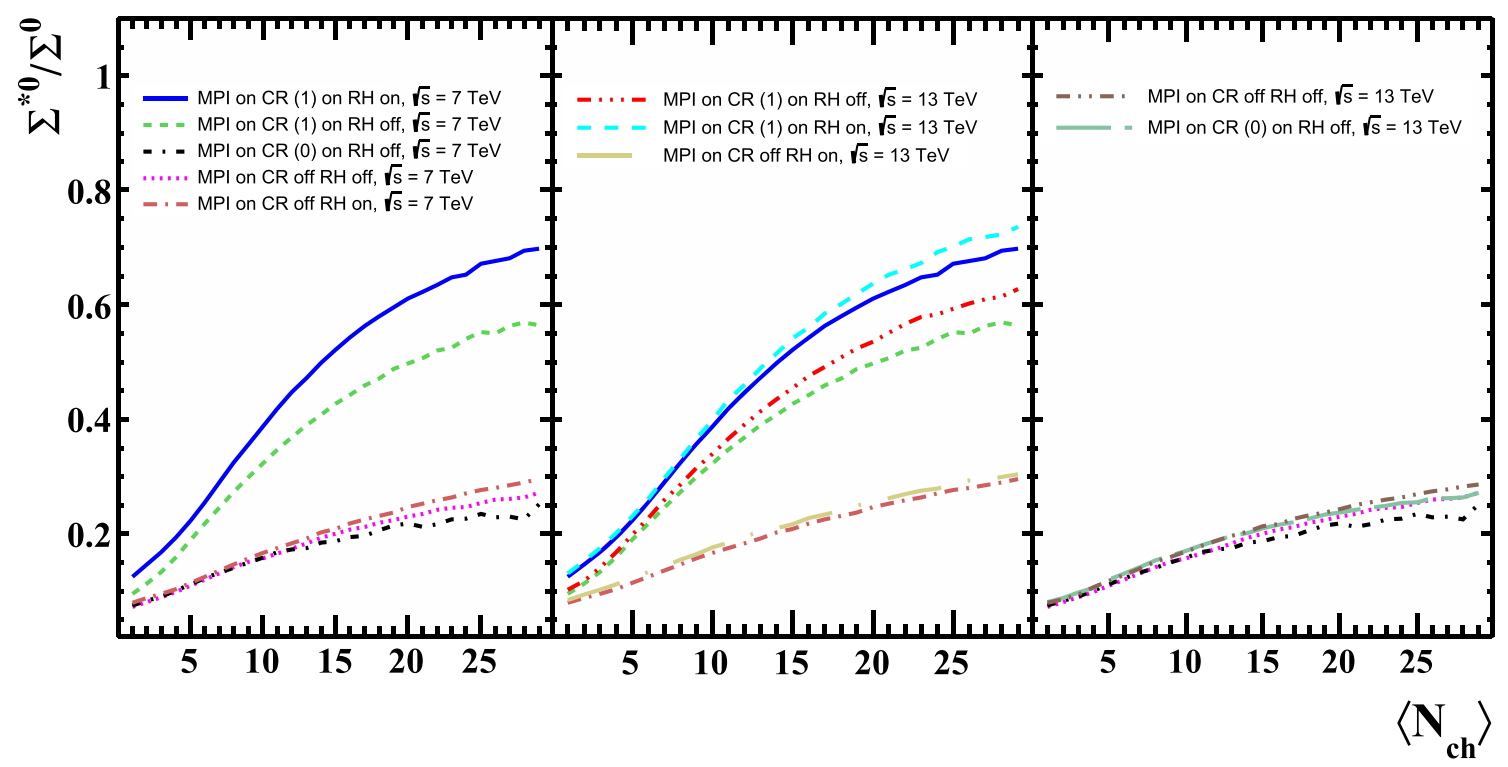

Fig. $7 p_{T}$-integrated ratio of $\Sigma^{0 *} / \Sigma$ as a function of $\left\langle N_{\text {ch }}>\right.$ in $\mathrm{p}-\mathrm{p}$ collisions. (Left panel) The ratios are compared for two different modes of CR [CR-off, $\mathrm{CR}(0)$ and $\mathrm{CR}(1)]$ with (and without) $\mathrm{RH}$ in $\mathrm{p}-\mathrm{p}$ collisions at $\sqrt{s}=7 \mathrm{TeV}$. (Middle panel) The ratios are compared for $\mathrm{p}-\mathrm{p}$ collisions at $\sqrt{s}=7 \mathrm{TeV}$ and $13 \mathrm{TeV}$ for CR-off, CR(0) and $\mathrm{CR}(1)$ with RH. (Right panel) Comparison of ratios for $\mathrm{p}-\mathrm{p}$ collisions at $\sqrt{s}=7 \mathrm{TeV}$ and $13 \mathrm{TeV}$ for two other modes, namely CR-off and $\mathrm{CR}(0)$ without $\mathrm{RH}$ 


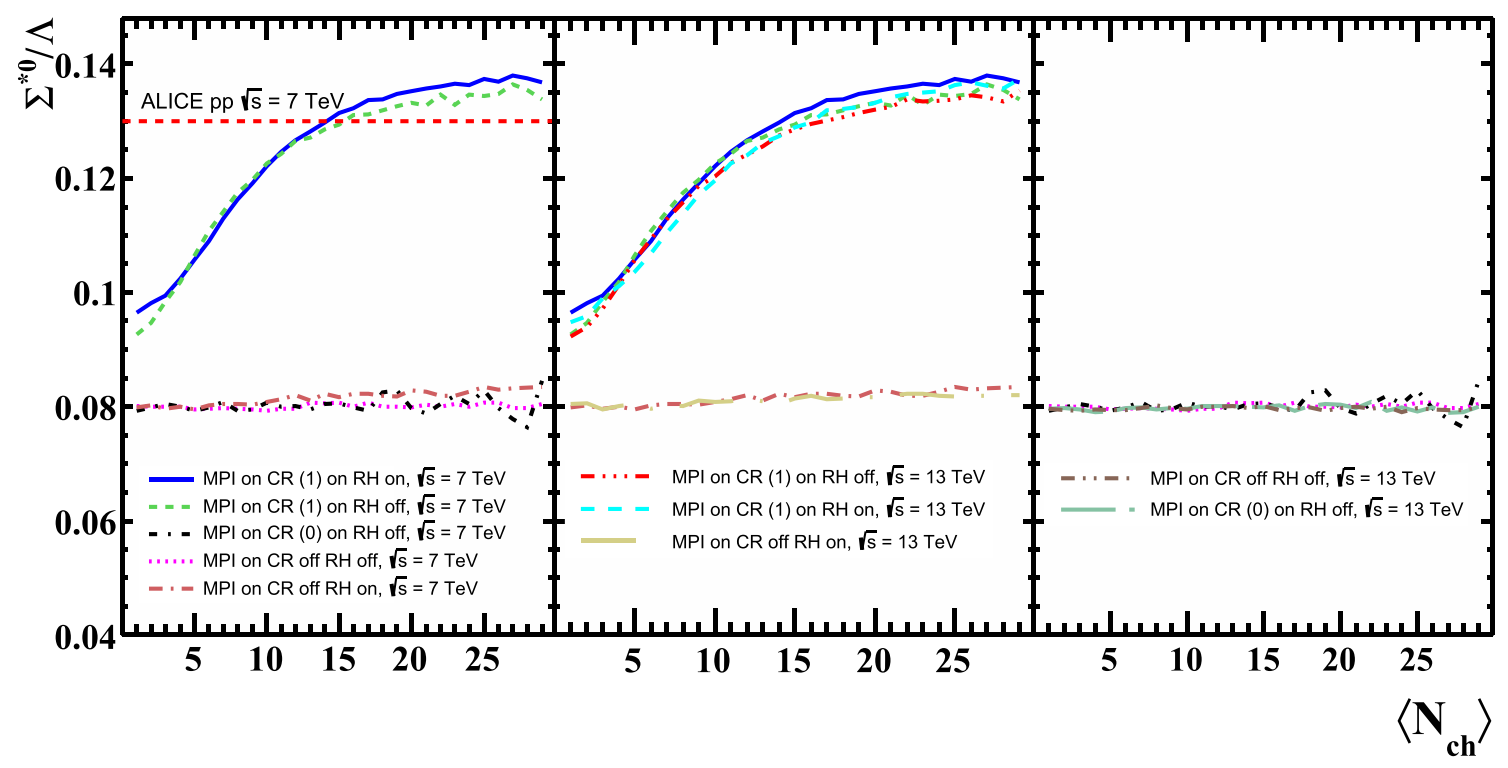

Fig. $8 p_{T}$-integrated ratio of $\Sigma^{0 *} / \Lambda$ as a function of $<N_{\text {ch }}>$ in $\mathrm{p}-\mathrm{p}$ collisions. (Left panel) The ratios are compared for two different modes of color reconnections [CR-off, $\mathrm{CR}(0)$ and $\mathrm{CR}(1)]$ with (and without) RH in $\mathrm{p}-\mathrm{p}$ collisions at $\sqrt{s}=7 \mathrm{TeV}$. (Middle panel) The ratios are compared for $\mathrm{p}-\mathrm{p}$ collisions at $\sqrt{s}=7 \mathrm{TeV}$ and $13 \mathrm{TeV}$ for CR-off,
$\mathrm{CR}(0)$ and $\mathrm{CR}(1)$ with RH. (Right panel) Comparison of ratios for $\mathrm{p}-\mathrm{p}$ collisions at $\sqrt{s}=7 \mathrm{TeV}$ and $13 \mathrm{TeV}$ for two other modes, namely $\mathrm{CR}$-off and $\mathrm{CR}(0)$ without $\mathrm{RH}$. The dashed line in the left panel shows the value of $\Sigma^{ \pm *} / \Lambda$ for minimum bias $\mathrm{p}-\mathrm{p}$ collisions at $\sqrt{s}=7 \mathrm{TeV}$ as obtained by ALICE experiment [23]

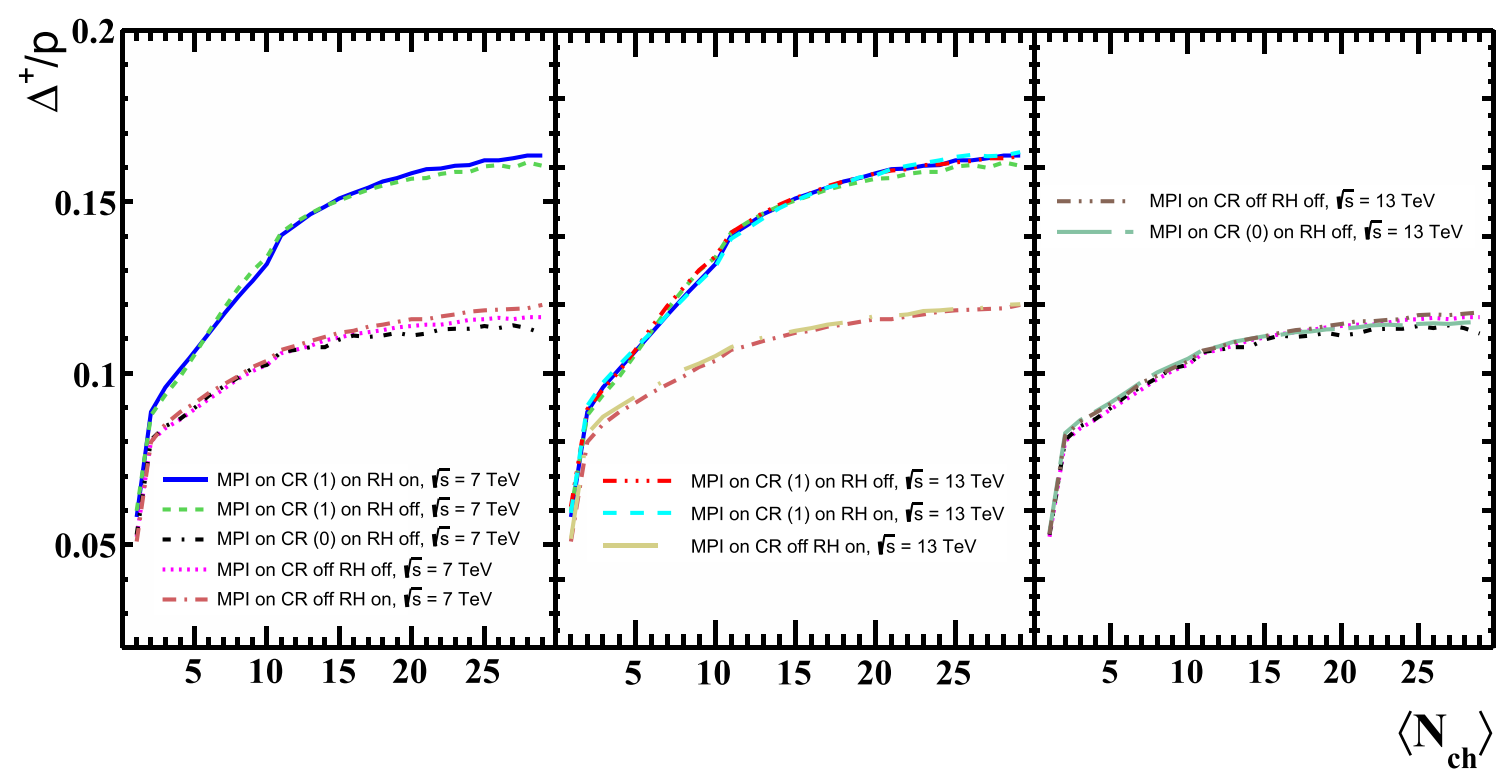

Fig. $9 p_{T}$-integrated ratio of $\Delta^{+} / p$ as a function of $<N_{\text {ch }}>$ in $\mathrm{p}-\mathrm{p}$ collisions. (Left panel) The ratios are compared for two different modes of color reconnections [CR-off, $\mathrm{CR}(0)$ and $\mathrm{CR}(1)]$ with (and without) $\mathrm{RH}$ in $\mathrm{p}-\mathrm{p}$ collisions at $\sqrt{s}=7 \mathrm{TeV}$. (Middle panel) The ratios are compared for $\mathrm{p}-\mathrm{p}$ collisions at $\sqrt{s}=7 \mathrm{TeV}$ and $13 \mathrm{TeV}$ for CR-off, $\mathrm{CR}(0)$ and $\mathrm{CR}(1)$ with RH. (Right panel) Comparison of ratios for $\mathrm{p}-\mathrm{p}$ collisions at $\sqrt{s}=7 \mathrm{TeV}$ and $13 \mathrm{TeV}$ for two other modes, namely CR-off and CR(0) without and provide an alternative description for suppression (or enhancement) of resonance-to-non-resonance ratios based on microscopic processes active in the partonic domain, and it additionally indicates that the presence of a thermalized medium is not imperative for the suppression of resonance- to-non-resonance particle ratios. It will also be interesting to study the resonance production mechanism using different QCD models, namely Herwig7.2 [24,25] and Sherpa2.2 [26]. These models include different underlying physics processes, parton shower, and hadronization mechanism, and 


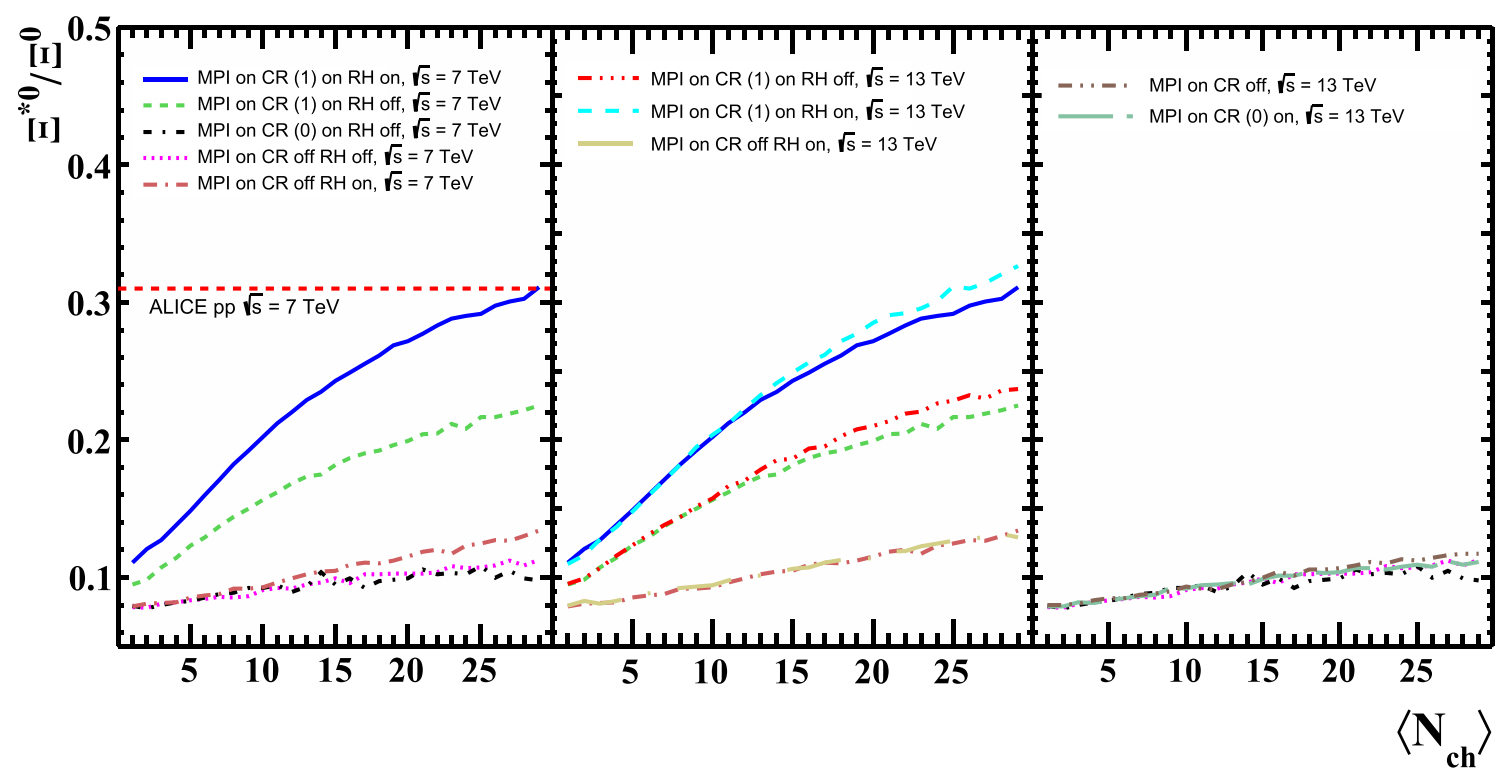

Fig. $10 p_{T}$-integrated ratio of $\Xi^{0 *} / \Xi^{0}$ as a function of $<N_{\mathrm{ch}}>$ in $\mathrm{p}-\mathrm{p}$ collisions. (Left panel) The ratios are compared for two different modes of color reconnections [CR-off, CR(0) and CR(1)] with (and without) RH in p-p collisions at $\sqrt{s}=7 \mathrm{TeV}$. (Middle panel) The ratios are compared for $\mathrm{p}-\mathrm{p}$ collisions at $\sqrt{s}=7 \mathrm{TeV}$ and $13 \mathrm{TeV}$ for

thus the study using these models is imperative for a clear understanding of resonance production.

\section{Summary}

The production of resonance particles in $\mathrm{p}-\mathrm{p}$ collisions was studied at $\sqrt{s}=7 \mathrm{TeV}$ and $13 \mathrm{TeV}$ using a Pythia 8 event generator. The ratio of the yield of resonance to non-resonance particles with similar quark content was obtained within the framework of microscopic processes including color reconnections and $\mathrm{RH}$. The ratios for various species of mesonic and baryonic resonances were studied as a function of the mean number of charged particles to study the effect of the considered microscopic processes. Suppression was observed for mesonic resonance ratios $\mathrm{K}^{* 0} / \mathrm{K}, \eta^{\prime} / \pi$, and $\rho^{0} / \pi$ for high event activity when the QCD-based color reconnection was enabled. This observation can be attributed to the formation of shorter strings which fragment into lowermass particles. The yield ratios for $\phi / \mathrm{K}$ and $\phi / \pi$ increased for QCD-based color reconnections and RH compared to other modes. The production of $\phi$ increased with the production of more strange (and anti-strange) quarks with color ropes. This observation is in agreement with the multiplicity dependence of $\phi / \mathrm{K}$ and $\phi / \pi$ as measured by the ALICE experiment, and the data favor the enhancement scenario due to color reconnections (1) and $\mathrm{RH}$ for the $\phi / \mathrm{K}$ and $\phi / \pi$ ratios. The model predicts an enhancement in the yield ratios for
CR-off, CR(0) and CR(1) with RH. (Right panel) Comparison of ratios for $\mathrm{p}-\mathrm{p}$ collisions at $\sqrt{s}=7 \mathrm{TeV}$ and $13 \mathrm{TeV}$ for two other modes, namely CR-off and $\mathrm{CR}(0)$ without $\mathrm{RH}$. The dashed line in the left panel shows the value of $\Xi^{0 *} / \Xi^{0}$ for minimum bias p-p collisions at $\sqrt{s}=7$ $\mathrm{TeV}$ as obtained by the ALICE experiment [23]

baryonic resonances. The ratios were found to be independent of the collision energy of the system. These predictions can serve as a baseline study for future measurements. The color reconnection and rope formation can explain part of the resonance suppression and enhancement. However, a more quantitative comparison is needed to confirm that the color reconnection and rope formation can be considered an alternative explanation of the observed resonance suppression and regeneration based on processes present in the partonic phase.

Acknowledgements The authors would like to thank the Department of Science and Technology (DST), India, for supporting the present work.

Data Availability Statement The manuscript has associated data in a data repository. [Authors' comment: The numerical values of the data points are extracted from the citation mentioned in the captions of each figure. The data from the published citation are available in HepData.]

Open Access This article is licensed under a Creative Commons Attribution 4.0 International License, which permits use, sharing, adaptation, distribution and reproduction in any medium or format, as long as you give appropriate credit to the original author(s) and the source, provide a link to the Creative Commons licence, and indicate if changes were made. The images or other third party material in this article are included in the article's Creative Commons licence, unless indicated otherwise in a credit line to the material. If material is not included in the article's Creative Commons licence and your intended use is not permitted by statutory regulation or exceeds the permitted use, you will need to obtain permission directly from the copyright holder. To view a copy of this licence, visit http://creativecomm 
ons.org/licenses/by/4.0/.

Funded by $\mathrm{SCOAP}^{3}$.

\section{References}

1. J. Adams et al. (ALICE Collaboration), Nat. Phys. 13, 535-539 (2017)

2. V. Khachatryan et al. (CMS Collaboration), JHEP 09, 091 (2010)

3. A. Ortiz Velasquez, P. Christiansen, E. Cuautle Flores, I.A. Maldonado Cervantes, G. Paic, Phys. Rev. Lett. 111, 042001 (2013)

4. C. Markert, R. Bellwied, I. Vitev, Phys. Lett. B 669, 92 (2008)

5. B.I. Abelev et al. (ALICE Collaboration), Phys. Rev. C 91, 024609 (2015)

6. M.M. Aggarwal et al. (STAR Collaboration), Phys. Rev. C 84, 034909 (2011)

7. S. Acharya et al. (ALICE Collaboration), Phys. Rev. C 99, 024905 (2019)

8. K. Werner, I. Karpenko, T. Pierog, M. Bleicher, K. Mikhailov, Phys. Rev. C 82, 044904 (2010)

9. A.G. Knospe, C. Markert, K. Werner, J. Steinheimer, M. Bleicher, Phys. Rev. C 93, 014911 (2016)

10. A.G. Knospe (ALICE Collaboration), J. Phys. Conf. Ser. 779, 012072 (2017)
11. R. Nayak, S. Pal, S. Dash, Phys. Rev. D 100, 074023 (2019)

12. C. Bierlich, Nucl. Phys. A 00, 1-5 (2018)

13. C. Bierlich, G. Gustafson, L. Lonnblad, MCnet-16-48, LU-TP 1664 (2016). arXiv:1612.05132 [hep-ph]

14. P. Chakraborty, S. Dash, Phys. Rev. C 102, 055202 (2020)

15. T. Sjostrand, S. Mrenna, P.Z. Skands, Comput. Phys. Commun. 178, 852 (2008)

16. R. Acconcia, D.D. Chinellato, R. Derradi de Souza, J. Takahashi, G. Torrieri, C. Markert, Phys. Rev. D 97, 036010 (2018)

17. J.R. Christiansen, P.Z. Skands, JHEP 1508 (2015)

18. C. Bierlich, J.R. Christiansen, Phys. Rev. D 92, 094010 (2015)

19. T.S. Biro, H.B. Nielson, J. Knoll, Nucl. Phys. B 245, 449 (1984)

20. C. Bierlich, G. Gustafson, L. Lonnblad, A. Tarasov, J. High Energy Phys. 2015, 148 (2015)

21. P. Skands, S. Carrazza, J. Rojo, Eur. Phys. J. C 74, 3024 (2014)

22. ALICE Collaboration, Phys. Lett. B 807, 135501 (2020). arXiv:1910.14397 [nucl-ex]

23. ALICE Collaboration, Nucl. Phys A, 1005, 121939 (2021). arXiv:2001.09023 [hep-ex]

24. J. Bellm, EPJ C 76(4), 196 (2016)

25. C.B. Duncan, P. Kirchgae $\beta$ er, EPJ C 79, 61 (2019)

26. E. Bothmann et al., SciPost Phys. 7, 034 (2019) 